OPEN ACCESS

Edited by:

Qianqian Su,

Shanghai University, China

Reviewed by:

Xiaowang Liu,

Northwestern Polytechnical

University, China

Qiuqiang Zhan,

South China Normal University, China

*Correspondence:

Lei Zhou

zhoul8@mail.sysu.edu.cn

Xian Chen

x.chen87@outlook.com

Specialty section:

This article was submitted to

Nanoscience,

a section of the journal

Frontiers in Chemistry

Received: 31 October 2020

Accepted: 21 December 2020

Published: 25 January 2021

Citation:

Sun T, Gao Y, Du Y, Zhou L and Chen X (2021) Recent Advances in Developing

Lanthanide Metal-Organic

Frameworks for Ratiometric

Fluorescent Sensing.

Front. Chem. 8:624592.

doi: $10.3389 /$ fchem.2020.624592

\section{Recent Advances in Developing Lanthanide Metal-Organic Frameworks for Ratiometric Fluorescent Sensing}

\author{
Tianying Sun ${ }^{1}$, Yaobin Gao ${ }^{1}$, Yangyang $\mathrm{Du}^{2}$, Lei Zhou ${ }^{1 *}$ and Xian Chen ${ }^{2 *}$ \\ ${ }^{1}$ School of Chemical Engineering and Technology/School of Marine Sciences, Sun Yat-sen University, Zhuhai, China, ${ }^{2}$ College of \\ Materials Science and Engineering, Shenzhen University, Shenzhen, China
}

Fluorescent probes have attracted special attention in developing optical sensor systems due to their reliable and rapid fluorescent response upon reaction with the analyte. Comparing to traditional fluorescent sensing systems that employ the intensity of only a single emission, ratiometric fluorescent sensors exhibit higher sensitivity and allow fast visual screening of analytes because of quantitatively analyzing analytes through the emission intensity ratio at two or more wavelengths. Lanthanide metal-organic frameworks (LnMOFs) are highly designable multifunctional luminescent materials as lanthanide ions, organic ligands, and guest metal ions or chromophores are all potential sources for luminescence. They thus have been widely employed as ratiometric fluorescent sensors. This mini review summarized the basic concept, optical features, construction strategies, and the ratiometric fluorescent sensing mechanisms of dual-emitting LnMOFs. The review ends with a discussion on the prospects, challenges, and new direction in designing LnMOF-based ratiometric fluorescent sensors.

Keywords: lanthanide, luminescence, dual emission, ratiometric sensing, metal-organic frameworks

\section{INTRODUCTION}

Fluorescent probes have attracted special attention in developing optical sensor systems due to their reliable and rapid fluorescent response upon reaction with the analyte (Vendrell et al., 2012). The strategy to develop effective fluorescent probes has become one of the hottest research areas in recent years. Various kinds of fluorescence probes based on organic dye molecules, semiconductor quantum dots (QDs) have been developed (Wu et al., 2020a; Yang et al., 2020a; Pfeifer et al., 2020). However, these probes have several limitations in detecting or sensing. For example, organic dyes suffer from several drawbacks such as poor chemical stability and rapid photobleaching, making it impossible for long-term fluorescence sensing. Compared with organic dye molecules, QDs exhibit better chemical and photostability. QDs are usually composed of heavy metals and suffer from toxicity and environmental hazards, which limit their practical applications. Lanthanide-based fluorescent probes can surpass the aforementioned inherent limitations of organic dyes or quantum dots due to their excellent properties, including low toxicity and better stability. Moreover, lanthanide luminescence features rich linelike emission bands, large Stokes shift, and high resistance to photobleaching, making the lanthanide-based luminescent materials superior in sensing applications. 
Traditional fluorescent sensing system that employs the intensity of only a single emission for quantitative analysis of analytes can be difficult and might yield unreliable results. A number of analyte-independent factors such as instrumental drift, the microenvironment and local concentration variance of probes, and photobleaching of the probes, all interfere with the quantification of the analyte and lower the sensing reliability. To circumvent these drawbacks, a ratiometric approach has been adopted in the design of fluorescent sensors (Chen et al., 2020; Zhao and Li, 2020). Ratiometric fluorescent sensors allow the simultaneous measurement of emission intensities at two or more wavelengths, and their emission intensity ratio is calculated and then correlated to analytes. In particular scenarios, change in emission color output could be observed upon reaction with the analyte, allowing for fast visual screening of analytes (Chen et al., 2018; Su et al., 2018; Bigdeli et al., 2019; Zhang et al., 2020). Perceiving the variation in the color brightness is much harder than visualizing the color change. Therefore, the ratiometric fluorescence sensing method is a clear winning strategy over the traditional single emission intensity-based sensing approach.

The search for novel lanthanide-based dual- or multi-emitting materials that suitable for developing a ratiometric sensor is currently an emerging field (Yin and Yin, 2020). Lanthanide metal-organic frameworks (LnMOFs) combine the lanthanide luminescent features with the MOFs' characteristics such as high porosity, large surface-to-volume ratio, diverse structures have been proved to be a powerful candidate in sensing application (Zhang et al., 2018; Liu et al., 2019). The permanent porosity and large surface area provide the potential to effectively concentrate analytes at higher levels within MOFs (Han et al., 2012), thus improve the sensitivity. Comparing to other conventional lanthanide hybrid platforms such as lanthanide-QDs or lanthanide-dye, LnMOFs present superior emission tuning capability as lanthanide nodes, organic linkers, the ligand to metal energy transfer, all of which can be "tailor-made" or modulated (Yan, 2017; Yang et al., 2020b; Wu et al., 2020c). Moreover, the intrinsic porosity of MOFs provides a pathway to encapsulate guest materials, which may also contribute to emission. Furthermore, the host framework-guest interactions such as coordination bonds, $\pi-\pi$ interaction, and hydrogen bonding provide excellent sensing sensitivity toward the analyte (Xu and Yan, 2017). Therefore, all these features make LnMOFs promising in developing ratiometric sensing systems.

In this mini review, we provide a general overview of the design principles of LnMOFs with multiple luminescent centers. To further discuss the details in design and the working mechanisms of these ratiometric fluorescent sensors, very recent progress in the development of LnMOFs for sensing ions, trace-water, gas and temperature, molecular decoding and biosensing will also be discussed. Our efforts here are to highlight the prospects of LnMOFs as ratiometric fluorescent sensors and briefly discuss the desired properties for future development.

\section{The Construction of Dual-Emitting LnMOFs}

The prerequisite of ratiometric assays is to generate dual or multi emission signals by one excitation. By virtue of the inherent hybrid nature, LnMOFs can mostly generate luminescent from the following component (Figure 1A): 1) Ligand usually conjugated organic compounds can generate emission upon excitation with UV or visible light. 2) Lanthanide metal nodes can give emission through antenna effect promoted by the ligands. 3) Lanthanide ions as guest species that incorporated into MOF structures could also yield luminescence. 4) Other chromophore guests, such as dye molecules or QDs. Figures 1B-D summarizes three typical scenarios by which dual emission has been generated and utilized for the construction of ratiometric sensor, namely a Ln (I)-Ln (II) bimetallic system, a Ln-Ligand system and a Ln-Chromophore system.

\section{Ln (I)-Ln (II) Bimetallic System in LnMOFs}

The traditional method of physically mixing two types of LnMOFs doped with different lanthanide ions, as illustrated in (i) of Figure 1B, is the most straightforward way to obtain dual emission (Chen et al., 2019). Due to the similar chemical properties of lanthanide ions, LnMOF structures can also allow for the incorporation of different types and ratios of lanthanide ions into the same host materials and thus generate characteristic emissions of each lanthanide ion at the same time ((ii) of Figure 1B) (Xia et al., 2017; Su et al., 2018; Ji et al., 2018; Gao et al., 2018; Li et al., 2019a; Othong et al., 2020; Zeng et al., 2020a). Once incorporated into the MOF structure, lanthanide ions can generate emission covering the spectrum region from ultraviolet to near-infrared. Among which, $\mathrm{Eu}^{3+}$ and $\mathrm{Tb}^{3+}$ ions exhibit strong red and green luminescence, respectively, that can be easily perceived by the naked eye. Therefore, $\mathrm{Eu}^{3+} / \mathrm{Tb}^{3+}$ based MOFs have been widely employed for the construction of dual emission ratiometric fluorescent sensor. For example, chemical fine-tuning the $\mathrm{Tb} / \mathrm{Eu}$ ratio of raw materials in the synthetic process of $\mathrm{Tb} / \mathrm{Eu}(\mathrm{TATB})$ results in tunable dual emission from $\mathrm{Tb}^{3+}$ and $\mathrm{Eu}^{3+}$ ions (Zeng et al., 2020a). Besides serving as the metal nodes, multiple types of lanthanide ions can also be encapsulated into the pore of MOFs as emissive guest species [(iii) of Figure 1B] (Li et al., 2019c; Qiao et al., 2019). For instance, Tb/Eu@bio-MOF-1 material was fabricated via ion exchange between $\mathrm{Tb}^{3+}$ and $\mathrm{Eu}^{3+}$ cations into the pores of the anionic framework, also resulting in dual emission from $\mathrm{Tb}^{3+}$ and $\mathrm{Eu}^{3+}$ ions (Zhang et al., 2016b). Noted that the porous microstructure around $\mathrm{Tb}^{3+}$ and $\mathrm{Eu}^{3+}$ ions also facilitate the absorption and the transportation of analyte and therefore contribute to the effective sensing.

\section{Ln-Ligand System in LnMOFs}

Luminescent behavior of LnMOFs is highly dependent on the efficiency of the antenna effect. The perfectly matched energy levels of ligand and metal result in emission from lanthanide ions dominantly, while poor energy matching leads to almost no lanthanide emission. In the case of partial energy transfer from the ligand to lanthanide ions, both characteristic emissions from lanthanide ion and ligand can be recorded. Such dual emissive Ln-Ligand LnMOF system can be helpful in constructing ratiometric sensors once exposed to analytes (Yang et al., 2017; Yue et al., 2018; Li et al., 2019b; Yin et al., 2019). For example, Qian et al. prepared ZJU-136-Ce $e_{1-x} \mathrm{Eu}_{\mathrm{x}}$ with 


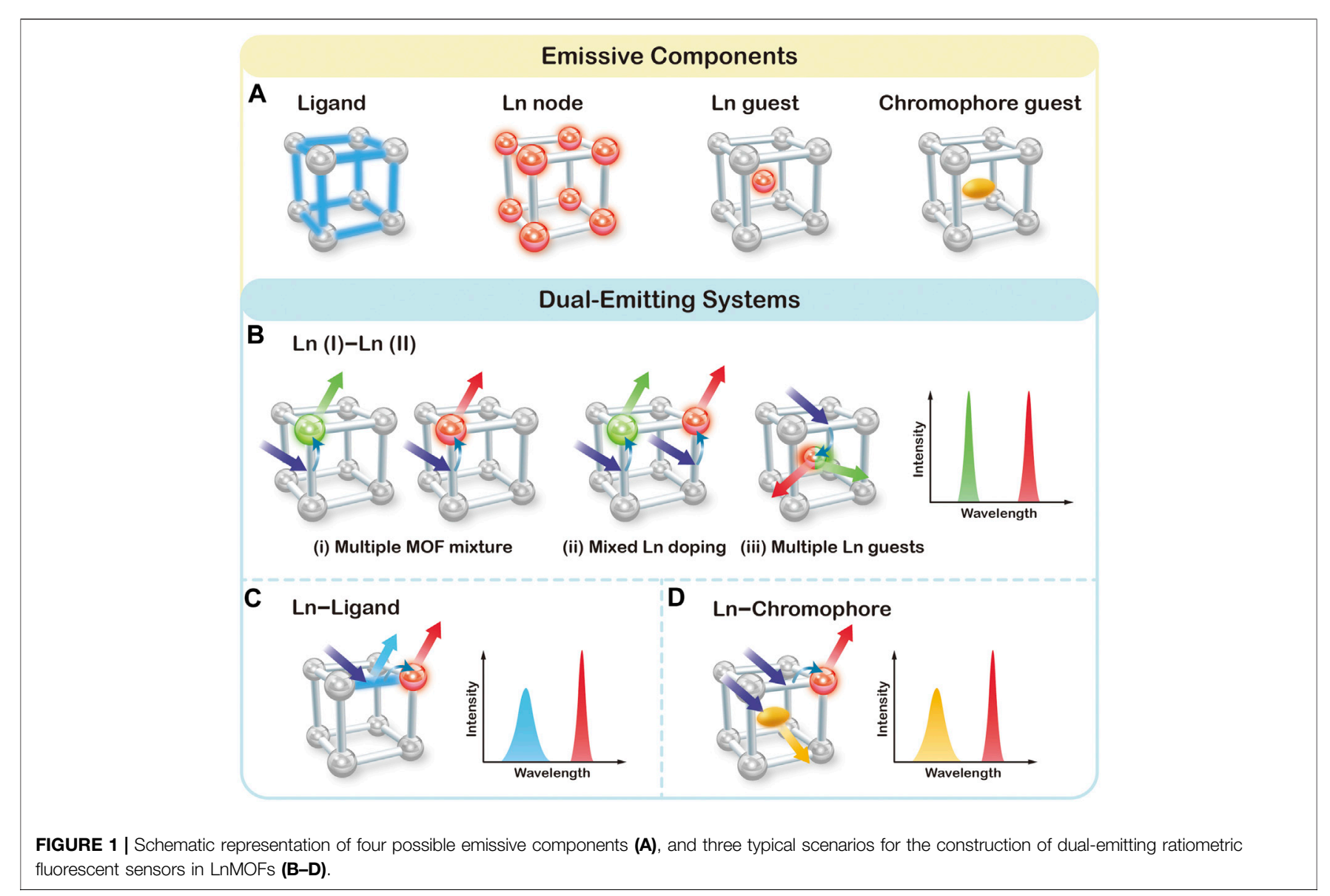

$1,1^{\prime} ; 4^{\prime}, 1^{\prime \prime}$-terphenyl-2',4,4", $5^{\prime}$-tetracarboxylic acid (TPTC) and $\mathrm{Ce}^{4+}$ and $\mathrm{Eu}^{3+}$ ions (Yue et al., 2018). ZJU-136-Ce ${ }_{1-\mathrm{x}} \mathrm{Eu}_{\mathrm{x}}$ exhibits dual emission from TPTC ligand at $390 \mathrm{~nm}$ and $\mathrm{Eu}^{3+}$ ions at $617 \mathrm{~nm}$ under $320 \mathrm{~nm}$ excitation. The introduced $\mathrm{Ce}^{4+}$ ions were intended to regulate the energy transfer from TPTC to $\mathrm{Eu}^{3+}$ ions. Due to the specific redox reaction between ascorbic acid (AA) and $\mathrm{Ce}^{4+}$, the emission intensity of TPTC increased significantly with the simultaneous quenching of $\mathrm{Eu}$ ions. Therefore, ZJU-136$\mathrm{Ce}_{1-x} \mathrm{Eu}_{x}$ was proved to be useful in ratiometric fluorescence sensing for AA determination.

\section{Ln-Chromophore System in LnMOFs}

MOFs possess pores and channels with highly tunable sizes and shapes. Apart from the intrinsic lanthanide luminescent from the frameworks and the encapsulated $\mathrm{Ln}^{3+}$ ions, there are plenty of chromophores such as metal-complex, organic dyes, QDs that can be encapsulated into MOF as chromophore guest (Cui et al., 2015; Gao et al., 2017; Hao et al., 2017; Qin and Yan, 2018; Wang et al., 2019; Yu et al., 2019). For instance, Yu and coworkers developed a water-stable RhB@Tb-dcpcpt host-guest composite by trapping the cationic rhodamine $\mathrm{B}(\mathrm{RhB})$ into the anionic framework of $\left[\mathrm{Me}_{2} \mathrm{NH}_{2}\right]\left[\mathrm{Tb}_{3}(\mathrm{dcpcpt})_{3}(\mathrm{HCOO})\right] \cdot \mathrm{DMF} \cdot 15 \mathrm{H}_{2} \mathrm{O}$ via an ion exchange process (Yu et al., 2019). The composite exhibit dual emission from $\mathrm{RhB}$ and $\mathrm{Tb}$ ions, and therefore allows the realization of sensitive and selective detection toward ciprofloxacin and norfloxacin antibiotics via a luminescent color-changing process. Wang et al. reported a dual-emitting carbon dots@Eu-MOFs for the ratiometric fluorescent detection of $\mathrm{Cr}(\mathrm{VI})$ (Wang et al., 2019). Upon reacting with $\mathrm{Cr}(\mathrm{VI})$, the fluorescence of carbon dots was quenched while the emission intensity of Eu-MOFs remains unchanged. Therefore, the prepared composites can be employed as self-calibrated probes for $\mathrm{Cr}(\mathrm{VI})$.

\section{Ratiometric Fluorescent Sensing Mechanisms}

In a dual-emitting ratiometric fluorescent sensing system, the sensor-analyte interaction can be categorized into the following: analyte modulates by either suppressing (i) or enhancing (ii) the energy transfer from the ligand to lanthanide ions, resulting in reduced or enhanced emission from lanthanide ions. (iii) Analyte modulates the coordination interaction of lanthanide ions. (iv) Analyte modulates the Ln-Ln energy transfer. In general, dualemitting properties of LnMOF-based sensors show a quick response once in reaction with analyte through the abovementioned interactions. And the intensity ratio of dual emission can be calculated and then correlated to analytes. In particular scenarios, changes in output emission color can be observed, allowing for fast visual screening of analytes. 


\section{Ratiometric Fluorescent Sensing Mechanisms}

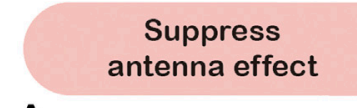

A

B
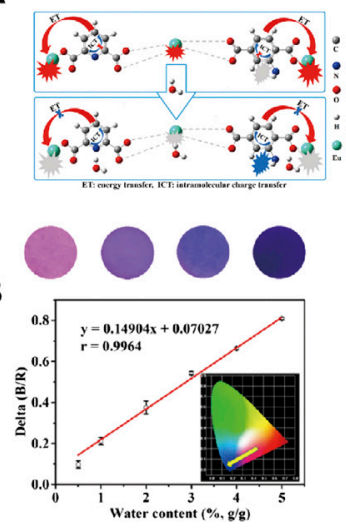

C

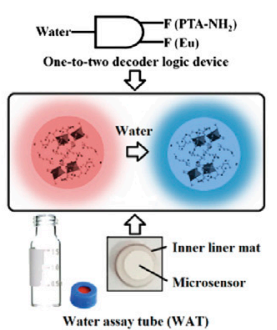

Enhance antenna effect

D

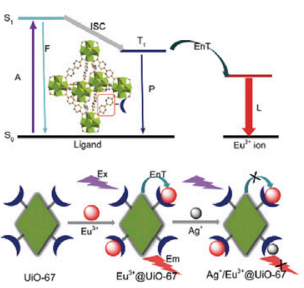

E

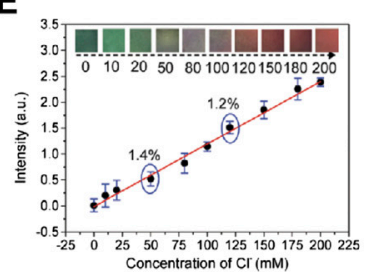

$\mathbf{F}$

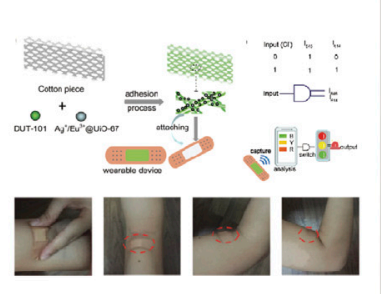

Change coordination environment of $\mathrm{Ln}$

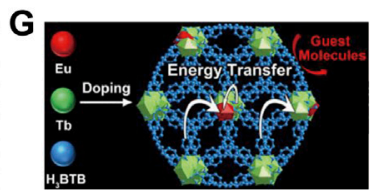

H

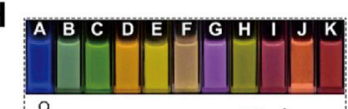

$\mathrm{N}_{\mathrm{OH}} \mathrm{\aleph OH}_{\mathrm{OH}} \mathrm{NH}^{\mathrm{NH}_{2}}, \mathrm{~N}$

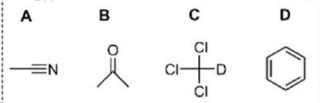

E $F \quad$ G

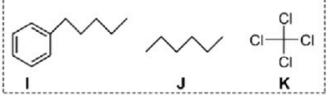

I

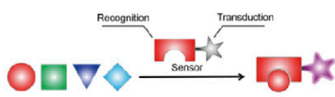

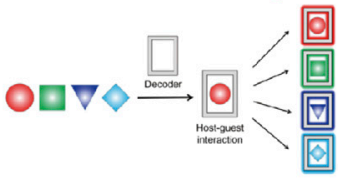

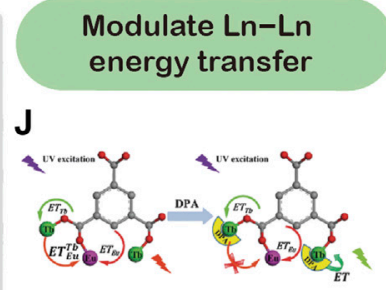

K

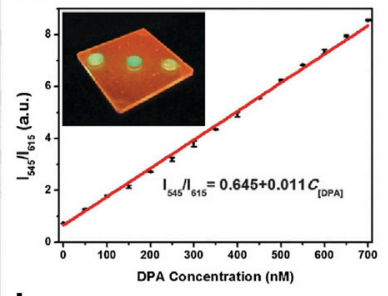

$\mathbf{L}$

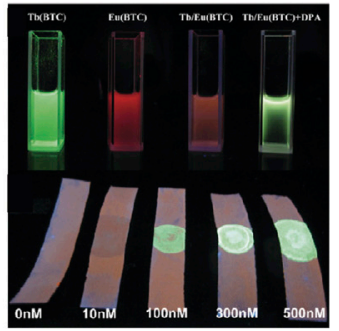

FIGURE 2 | In a dual-emitting system, analyte can be probed through the four commonly employed mechanisms. Analyte interacts with the sensor by suppressing (A-C) or enhancing (D-F) the antenna effect. Reproduced from Yu et al. (2020) with permission from American Chemical Society and Zhang et al. (2018) with permission from The Royal Society of Chemistry. The analyte may also change the coordination environment of Ln (G,H) or modulate the lanthanide-lanthanide energy transfer (I,J). Reproduced from Zeng et al. (2020a) with permission from American Chemical Society and Zhang et al. (2016a) with permission from The Royal Society of Chemistry.

The modulation of antenna effect to quench the lanthanide luminescence is probably the most commonly employed sensing approach by LnMOFs (Cui et al., 2019; Moscoso et al., 2020; Yu et al., 2020). For example, Yu et al. (2020) developed a trace water sensor based on Eu-MOF with the mixed ligand of dipicolinic acid (DPA) and 2-aminophthalic acid (PTA-NH ${ }_{2}$ ). DPA functioned as the sensitizer for $\mathrm{Eu}^{3+}$ ions, while PTA- $\mathrm{NH}_{2}$ provides a second emission besides $\mathrm{Eu}^{3+}$. The exposure of DPA to water leads to the occurrences of efficient intramolecular charge transfer (ICT) in DPA, which weakens the antenna effect from DPA to $\mathrm{Eu}^{3+}$ (Figure 2A). And they observed a "turn-on" blue emission of PTA-NH $\mathrm{N}_{2}$ with the water content in the MOF and a "turn-off" red emission from $\mathrm{Eu}^{3+}$ under $254 \mathrm{~nm}$ UV light excitation (Figure 2B). The increase of PTA- $\mathrm{NH}_{2}$ blue emission was ascribed to the waterinduced ICT fluorescence of PTA- $\mathrm{NH}_{2}$. This ratiometric fluorescent sensor exhibits a linear and sensitive response to water in the range of $0-100 \%(\mathrm{v} / \mathrm{v})$. Therefore, a one-to-two decoder logic device for water assay can be designed
(Figure 2C). Based on this dual-emitting ratiometric sensing technique, a paper-based water microsensor was fabricated for portable, longer-term stable, and rapid sensing. In 2017, Yang et al. developed a boric acid functional Eu-MOF probe for the recognition of fluoride ion, which is also based on the suppressed antenna effect (Yang et al., 2017). The insufficient ICT from ligand to $\mathrm{Eu}^{3+}$ results in the dual emission from ligand at $366 \mathrm{~nm}$ and $\mathrm{Eu}^{3+}$ ions at $625 \mathrm{~nm}$ under $275 \mathrm{~nm}$ excitation. With the presence of fluoride ions, the binding of fluoride to the boron center disrupted the $\mathrm{p}_{\pi}-\pi$ conjugation of 5-bop and decreased the intersystem crossing efficiency, and therefore enhance the ligand emission while decreasing the $\mathrm{Eu}^{3+}$ emission with increased fluoride concentration. An excellent linear relationship can be observed between the intensity ratio of the dual emission and fluoride concentration in the range from 4 to $80 \mu \mathrm{M}$. Moreover, the boric acid exhibits a strong affinity to fluoride ions, while its interaction with other anions is relatively weak, making it perfect for fluoride selective sensing. 
Reports on enhancing antenna effect resulted in "turn-on" sensing are rather few comparing to the "turn-off" mechanism discussed above (Karmakar et al., 2019; Fueyo-González et al., 2020; Min et al., 2020; Wang et al., 2020; Yue et al., 2020). However, it is obvious that "turn-on" sensors have advantages over the "turn-off" sensors, especially in the quantitative determination of an analyte in low concentration (Luo et al., 2020). For the "turn-off" sensor, the signal decrease against an already bright background makes it hard for sensitive and accurate sensing. By contrast, "turn-on" luminescent behavior can significantly enhance a weak emission or even create a new emission, reducing the likelihood of false-positive signals while providing much higher sensitivity. Yang et al. developed a "turnon" ratiometric fluorescent sensor for $\mathrm{H}_{2} \mathrm{~S}$ by encapsulating $\mathrm{Tb}^{3+}$ ions within the pores of $\mathrm{MOF}\left[\mathrm{Cu}(\mathrm{HCPOC})_{2}\right] n$ to form $\mathrm{Tb}^{3+} @$ $\mathrm{Cu}-\mathrm{MOF}$ (Zheng et al., 2017). The composite exhibits weak $\mathrm{Tb}^{3+}$ emission and strong $\mathrm{H}_{2}$ CPOC ligand emission because the unsaturated electronic state of $\mathrm{Cu}^{2+}$ tends to have a negative effect on the energy transfer from ligand to $\mathrm{Tb}^{3+}$ ions. Serving as a strong electron donor, $\mathrm{H}_{2} \mathrm{~S}$ can decrease the binding energies of the metal center through its strong affinity for $\mathrm{Cu}^{2+}$ ions. Therefore, the luminescence of $\mathrm{Tb}^{3+}$ recovered as the result of the restored antenna effect between ligand and $\mathrm{Tb}^{3+}$. Meanwhile, the ligand showed a slight decrease in emission with the increase of $\mathrm{H}_{2} \mathrm{~S}$ concentration. Therefore, $\mathrm{Tb}^{3+} @ \mathrm{Cu}-\mathrm{MOF}$ sensor showed excellent sensitivity and selectivity toward $\mathrm{H}_{2} \mathrm{~S}$ with a linear response range from 13.25 to $1.6 \mathrm{mM}$. Another example of enhancing antenna effect-based ratiometric sensing was proposed by $\mathrm{Xu}$ and Yan, 2018. They developed a sweat $\mathrm{Cl}^{-}$ sensor based on $\mathrm{Ag}^{+} / \mathrm{Eu}^{3+} @ \mathrm{UiO}-67$ platform. As shown in Figure $2 \mathrm{D}, \mathrm{Ag}^{+}$was deliberately introduced into the system via a $\mathrm{Ag}-\mathrm{N}$ coordination bond to reduce the efficiency of the energy transfer from $\mathrm{UiO}-67$ to $\mathrm{Eu}^{3+}$. Upon reaction with $\mathrm{Cl}^{-}$, the strong electronic affinity between $\mathrm{Ag}^{+}$and $\mathrm{Cl}^{-}$leads to a "turnon" luminescence of $\mathrm{Eu}^{3+}$. By employing DUT-101 as a reference signal, an obvious output color shift from dark green to bright red can be observed, and a $0.1 \mathrm{mM} \mathrm{Cl}^{-}$limit of detection was claimed (Figure 2E). They further load the two LnMOF-based sensors to a cotton piece and attach it to a band-aid for the demonstration of a wearable $\mathrm{Cl}^{-}$monitoring device (Figure 2F).

Molecules with different coordination capability could cause changes in the coordination environment of lanthanide ions in MOF structures and further affect their emission (Cui et al., 2014; Zeng et al., 2020b). Based on this phenomenon, Zeng et al. developed fast and facile decoding of a broad range of molecules, including homologues, isomers, enantiomers, and deuterated isotopomers by employing $\mathrm{Tb} / \mathrm{Eu}(\mathrm{BTB})$ as the probe (Zeng et al., 2020b). 1,3,5-benzenetrisbenzoic acid $\left(\mathrm{H}_{3} \mathrm{BTB}\right)$ was chosen as the ligand, together with mixed $\mathrm{Tb}^{3+}$ and $\mathrm{Eu}^{3+}$, to construct $\mathrm{Tb} / \mathrm{Eu}(\mathrm{BTB})$ probe by a simple one-pot synthesis procedure (Figure 2G). Under $302 \mathrm{~nm}$ UV excitation, the probe exhibits red $\left(\mathrm{Eu}^{3+}\right)$, green $\left(\mathrm{Tb}^{3+}\right)$, and blue emissions (BTB) in a single host. Therefore this probe should be ideal for molecular decoding as it involves wide coverage in the visible spectral range. They found out that the ultrahigh decoding capability was realized by fine-tuning the energy transfer pathway in multiple ways, including changing the coordination environment of lanthanide ions within $\mathrm{Tb} /$ $\mathrm{Eu}(\mathrm{BTB})$ probe. For example, molecular with high coordination capability such as acetic acid could destroy the crystalline structure of LnMOFs and further eliminate the antenna effect. The binding of target molecules to $\mathrm{Eu}^{3+} / \mathrm{Tb}^{3+}$ could change the coordination symmetry of the LnMOFs and modulate the energy of singlet or triplet excited states of BTB, affecting the energy transfer from $\mathrm{BTB}$ to $\mathrm{Eu}^{3+} / \mathrm{Tb}^{3+}$. As shown in Figure $\mathbf{2 H}$, this ratiometric molecular decoding strategy enables a full-color readout and visual decoding capability. Conventional molecular decoding strategy requires a tailor-designed probe for a specific sensing target, which is time-consuming and laborintensive. The reported probes can accommodate a diverse range of molecules, while each gives a different output signal as the result of specific host-guest interaction (Figure 2I). This decoding strategy allows for multiple-target differentiation by using only a single probe, significantly promoting its widespread usability.

Modulating the Ln-Ln energy transfer is a straightforward way to tune the emission ratio of two lanthanide ions and correlate with the concentration of analytes (Zhang et al., 2016a; Zhao et al., 2019; Wu et al., 2020b; Feng et al., 2020). For example, Zhang and coworkers developed a dual-emitting $\mathrm{Tb} / \mathrm{Eu}(\mathrm{BTC})$ probe for the rapid detection of dipicolinic acid (DPA) biomarker (Zhang et al., 2016a). Since DPA can chelate with $\mathrm{Tb}^{3+}$, the energy transfer from DPA to $\mathrm{Tb}^{3+}$ becomes dominant while the Tb-to-Eu energy transfer is interrupted (Figure 2J). The concentration of DPA can be linearly correlated well with the ratio $I_{545} / I_{613}$ in the 50-700 nM concentration range with the estimated detection limit of $4.55 \mathrm{nM}$ (Figure 2K). And the color output of $\mathrm{Tb} /$ $\mathrm{Eu}(\mathrm{BTC})$ sensor changes from orange-red to yellow-green after adding DPA in the solution or dropping onto test strips (Figure 2L). Another great example of using an analyte to modulate Ln-Ln energy transfer is for ratiometric temperature sensing (Yang et al., 2020c; Feng et al., 2020). Feng et al. developed $\mathrm{Eu}_{0.0025} \mathrm{~Tb}_{0.9975}$-BABDC-PBMA membrane. $\mathrm{H}_{2}$ BABDC is an ideal "antenna chromophore" to sensitize both $\mathrm{Eu}^{3+}$ and $\mathrm{Tb}^{3+}$, and therefore generate dual emission (Feng et al., 2020). They found that the intensity ratio of ${ }^{5} \mathrm{D}_{4} \rightarrow{ }^{7} \mathrm{~F}_{5}\left(\mathrm{~Tb}^{3+}\right) /{ }^{5} \mathrm{D}_{0} \rightarrow{ }^{7} \mathrm{~F}_{2}\left(\mathrm{Eu}^{3+}\right)$ can be linear related to the temperature from 90 to $240 \mathrm{~K}$. And the energy transfer from $\mathrm{Tb}^{3+}$ to $\mathrm{Eu}^{3+}$ was responsible for the unique temperature-dependent luminescent behavior. Benefiting from the tunable architectures of this probe, they then developed a free-standing and homogeneous membranous ratiometric luminescent thermometer with excellent mechanical properties. And the thermometer was tested to be stable even under harsh conditions, pushing forward the real application of LnMOF-based thermometers.

\section{CONCLUSIONS AND PERSPECTIVE}

This mini review provides a brief overview of the strategies to construct a dual-emitting LnMOF-based ratiometric fluorescent sensor. By virtue of the structural versatility, luminescent turnability, unique sensor-analyte interaction, and the possible fabrication into smart-devices make LnMOF an excellent 
candidate for efficient ratiometric fluorescent sensing. This technique relies on the change in the emission intensity of two emission bands, allowing for precise, quantitative, and real-time analysis. And the direct witness of the color change by the naked eye could be a more facile way for the determination of analytes. Despite the undeniable merits of LnMOF-based ratiometric fluorescent sensors, there are still many challenges to overcome. The stability, especially the water stability of the LnMOF-based sensors, needs to be further enhanced to avoid the structural breakdown before completing the sensing in the aqueous environment or other sensing media. Most of the LnMOFs sensing experiments were still conducted in solution. The development of LnMOF films or membranes as portable devices while maintaining the original stability and ratiometric sensing capability can be meaningful during the exploration of their practical applications. The design and fabrication of LnMOFs in nanometer scale is still in their infancy. Further effort can be devoted to preparing nanostructured LnMOFs,

\section{REFERENCES}

Bigdeli, A., Ghasemi, F., Abbasi-Moayed, S., Shahrajabian, M., Fahimi-Kashani, N., Jafarinejad, S., et al. (2019). Ratiometric fluorescent nanoprobes for visual detection: design principles and recent advances-a review. Anal. Chim. Acta. 1079, 30-58. doi:10.1016/j.aca.2019.06.035

Chen, D. H., Haldar, R., Neumeier, B. L., Fu, Z. H., Feldmann, C., Wöll, C., et al. (2019). Tunable emission in heteroepitaxial Ln-SURMOFs. Adv. Funct. Mater. 29, 1903086. doi:10.1002/adfm.201903086

Chen, D. M., Sun, C. X., Peng, Y., Zhang, N. N., Si, H. H., Liu, C. S., et al. (2018). Ratiometric fluorescence sensing and colorimetric decoding methanol by a bimetallic lanthanide-organic framework. Sensor. Actuator. B. Chem. 265, 104-109. doi:10.1016/j.snb.2018.03.028

Chen, L., Liu, D. H., Peng, J., Du, Q. Z., and He, H. (2020). Ratiometric fluorescence sensing of metal-organic frameworks: tactics and perspectives. Coord. Chem. Rev. 404, 213113. doi:10.1016/j.ccr.2019.213113

Cui, Y., Chen, F., and Yin, X. B. (2019). A ratiometric fluorescence platform based on boric-acid-functional Eu-MOF for sensitive detection of $\mathrm{H}$. Biosens. Bioelectron. 135, 208-215. doi:10.1016/j.bios.2019.04.008

Cui, Y., Song, R., Yu, J., Liu, M., Wang, Z., Wu, C., et al. (2015). Dual-emitting MOFdye composite for ratiometric temperature sensing. Adv. Mater. Weinheim. 27, 1420-1425. doi:10.1002/adma.201404700

Cui, Y. J., Chen, B. L., and Qian, G. D. (2014). Lanthanide metal-organic frameworks for luminescent sensing and light-emitting applications. Coord. Chem. Rev. 273-274, 76-86. doi:10.1016/j.ccr.2013.10.023

Feng, T. T., Ye, Y. X., Liu, X., Cui, H., Li, Z. Q., Zhang, Y., et al. (2020). A robust mixed-lanthanide polyMOF membrane for ratiometric temperature sensing. Angew. Chem. Int. Ed. 59, 2-8. doi:10.1002/anie.202009765

Fueyo-González, F., Garcia-Fernandez, E., Martínez, D., Infantes, L., Orte, A., González-Vera, J. A., et al. (2020). Smart lanthanide antennas for sensing water. ChemComm. 56, 5484-5487. doi:10.1039/d0cc01725f

Gao, J., Li, Q., Wang, C. H., and Tan, H. L. (2017). Copper (II)-mediated fluorescence of lanthanide coordination polymers doped with carbon dots for ratiometric detection of hydrogen sulfide. Sensor. Actuator. B. Chem. 253, 27-33. doi:10.1016/j.snb.2017.06.092

Gao, Y. X., Yu, G., Liu, K., and Wang, B. (2018). Luminescent mixed-crystal LnMOF thin film for the recognition and detection of pharmaceuticals. Sensor. Actuator. B. Chem. 257, 931-935. doi:10.1016/j.snb.2017.10.180

Han, S. B., Hermans, T. M., Fuller, P. E., Wei, Y. H., and Grzybowski, B. A. (2012). Transport into metal-organic frameworks from solution is not purely diffusive. Angew. Chem. Int. Ed. 51, 2662-2666. doi:10.1002/anie. 201108492

Hao, J., Liu, F. F., Liu, N., Zeng, M. L., Song, Y. H., and Wang, L. (2017). Ratiometric fluorescent detection of $\mathrm{Cu}^{2+}$ with carbon dots chelated which holds great promise in bioimaging, diagnosis, and therapy. Most of the LnMOF-based sensors are still based on the quenching mechanism. Research endeavors are preferred to devote toward the rational design of "turn-on" especially "turnon" ratiometric fluorescent sensors.

\section{AUTHOR CONTRIBUTIONS}

All authors listed have made a substantial, direct, and intellectual contribution to the work and approved it for publication.

\section{FUNDING}

This work was supported by the National Natural Science Foundation of China (Nos. 51802198 and 51902355), the start-up Grant of Sun Yat-sen University.

Eu-based metal-organic frameworks. Sensor. Actuator. B. Chem. 245, 641-647. doi:10.1016/j.snb.2017.02.029

Ji, G. F., Wang, J. Z., Gao, X. C., Liu, J. J., Guan, W. H., Liu, H. T., et al. (2018). Hypersensitive self-referencing detection traces of water in ethyl alcohol by dual-emission lanthanide metal-organic frameworks. Eur. J. Inorg. Chem. 14, 1998-2003. doi:10.1002/ejic.201800012

Karmakar, A., Samanta, P., Dutta, S., and Ghosh, S. K. (2019). Fluorescent "turnon" sensing based on metal-organic frameworks (MOFs). Chem. Asian J. 14, 4506-4519. doi:10.1002/asia.201901168

Li, B. B., Wang, W. J., Hong, Z. X., El-Sayed, E. S. M., and Yuan, D. Q. (2019a). Ratiometric fluorescence detection of trace water in an organic solvent based on bimetallic lanthanide metal-organic frameworks. Chem Comm. 55, 6926-6929. doi: $10.1039 / \mathrm{c} 9 \mathrm{cc} 02324 \mathrm{k}$

Li, H., Han, W., Lv, R., Zhai, A., Li, X. L., Gu, W., et al. (2019b). Dual-function mixed-lanthanide metal-organic framework for ratiometric water detection in bioethanol and temperature sensing. Anal. Chem. 91, 2148-2154. doi:10.1021/ acs.analchem. 8 b0 4690

Li, H. J., Li, Q. Q., and Xu, Z. Q. (2019c). Lanthanide cation encapsulated in a metal-organic framework as a white LED and selective naked-eye reversible $\mathrm{HCl}$ sensor. J. Mater. Chem. C. 7, 2880-2885. doi:10.1039/c8tc05956j

Liu, Y., Xie, X. Y., Cheng, C., Shao, Z. S., and Wang, H. S. (2019). Strategies to fabricate metal-organic framework(MOF)-based luminescent sensing platforms. J. Mater. Chem. C. 7, 10743-10763. doi:10.1039/c9tc03208h

Luo, T. Y., Das, P., White, D. L., Liu, C., Star, A., and Rosi, N. L. (2020). Luminescence "turn-on" detection of gossypol using $\mathrm{Ln}^{3+}$-based metal-organic frameworks and $\mathrm{Ln}^{3+}$ salts. J. Am. Chem. Soc. 142, 2897-2904. doi:10.1021/jacs.9b11429

Min, H., Han, Z. S., Wang, M. M., Li, Y. J., Zhou, T. Z., Shi, W., et al. (2020). A water-stable terbium metal-organic framework as a highly sensitive fluorescent sensor for nitrite. Inorg. Chem. Front. 12, 33-39. doi:10.1039/d0qi00780c

Moscoso, F. G., Almeida, J., Sousaraei, A., Lopes-Costa, T., Silva, A. M. G., Cabanillas-Gonzalez, J., et al. (2020). A lanthanide MOF immobilized in PMMA transparent films as a selective fluorescence sensor for nitroaromatic explosive vapours. J. Mater. Chem. C. 8, 3626-3630. doi:10.1039/d0tc00376j

Othong, J., Boonmak, J., Kielar, F., Hadsadee, S., Jungsuttiwong, S., and Youngme, S. (2020). Self-calibrating sensor with logic gate operation for anthrax biomarker based on nanoscaled bimetallic lanthanoid MOF. Sensor. Actuator. B. Chem. 316, 128156. doi:10.1016/j.snb.2020.128156

Pfeifer, D., Russegger, A., Klimant, I., and Borisov, S. M. (2020). Green to red emitting BODIPY dyes for fluorescent sensing and imaging of carbon dioxide. Sensor. Actuator. B. Chem. 304, 127312. doi:10.1016/j.snb.2019.127312

Qiao, X. F., Han, Y. B., Tian, D., Yang, Z. C., Li, J. L., and Zhao, S. T. (2019). MOF matrix doped with rare earth ions to realize ratiometric fluorescent sensing of 2,4,6-trinitrophenol: synthesis, characterization and performance. Sensor. Actuator. B. Chem. 286, 1-8. doi:10.1016/j.snb.2019.01.111 
Qin, S. J., and Yan, B. (2018). Dual-emissive ratiometric fluorescent probe based on $\mathrm{Eu}^{3+} / \mathrm{C}$-dots@MOF hybrids for the biomarker diaminotoluene sensing. Sensor. Actuator. B. Chem. 272, 510-517. doi:10.1016/j.snb.2018.06.018

Su, Y., Yu, J. H., Li, Y. B., Phua, S. F. Z., Liu, G. F., Lim, W. Q., et al. (2018). Versatile bimetallic lanthanide metal-organic frameworks for tunable emission and efficient fluorescence sensing. Commun. Chem. 1, 1-13. doi:10.1038/s42004018-0016-0

Vendrell, M., Zhai, D. T., Er, J. C., and Chang, Y. T. (2012). Combinatorial strategies in fluorescent probe development. Chem. Rev. 112, 4391-4420. doi:10.1021/cr200355j

Wang, H., Wang, X. L., Liang, M. S., Chen, G., Kong, R. M., Xia, L., et al. (2020). A boric acid-functionalized lanthanide metal-organic framework as a fluorescence "turn-on" probe for selective monitoring of $\mathrm{Hg}^{2+}$ and $\mathrm{CH}_{3} \mathrm{Hg}^{+}$. Anal. Chem. 92, 3366-3372. doi:10.1021/acs.analchem.9b05410

Wang, Y. Y., He, J., Zheng, M. D., Qin, M. D., and Wei, W. (2019). Dual-emission of Eu based metal-organic frameworks hybrids with carbon dots for ratiometric fluorescent detection of $\mathrm{Cr}(\mathrm{VI})$. Talanta. 191, 519-525. doi:10.1016/j.talanta. 2018.08.078

Wu, L. L., Huang, C. S., Emery, B. P., Sedgwick, A. C., Bull, S. D., He, X. P., et al. (2020a). Förster resonance energy transfer (FRET)-based small-molecule sensors and imaging agents. Chem. Soc. Rev. 49, 5110-5139. doi:10.1039/ c9cs00318e

Wu, M. N., Zhuang, Y. X., Liu, J. B., Chen, W. W., Li, X. Y., and Xie, R. J. (2020b). Ratiometric fluorescence detection of 2,6-pyridine dicarboxylic acid with a dual-emitting lanthanide metal-organic framework (MOF). Opt. Mater. 106, 110006. doi:10.1016/j.optmat.2020.110006

Wu, S. Y., Min, H., Shi, W., and Cheng, P. (2020c). Multicenter metal-organic framework-based ratiometric fluorescent sensors. Adv. Mater. 32, 1805871. doi:10.1002/adma.201805871

Xia, T. F., Cui, Y. J., Yang, Y., and Qian, G. D. (2017). Highly stable mixedlanthanide metal-organic frameworks for self-referencing and colorimetric luminescent $\mathrm{pH}$ sensing. ChemNanoMat. 3, 51-57. doi:10.1002/cnma. 201600331

Xu, X. Y., and Yan, B. (2018). A fluorescent wearable platform for sweat $\mathrm{Cl}^{-}$ analysis and logic smart-device fabrication based on color adjustable lanthanide MOFs. J. Mater. Chem. 6, 1863-1869. doi:10.1039/c7tc05204a

$\mathrm{Xu}, \mathrm{X}$. Y., and Yan, B. (2017). Intelligent molecular searcher from logic computing network based on $\mathrm{Eu}(\mathrm{III})$ functionalized UMOFs for environmental monitoring. Adv. Funct. Mater. 27, 1700247. doi:10.1002/adfm.201700247

Yan, B. (2017). Lanthanide-functionalized metal-organic framework hybrid systems to create multiple luminescent centers for chemical sensing. Acc. Chem. Res. 50, 2789-2798. doi:10.1021/acs.accounts.7b00387

Yang, G. H., Zhao, J. L., Yi, S. Z., Wan, X. J., and Tang, J. N. (2020a). Biodegradable and photostable $\mathrm{Nb}_{2} \mathrm{C}$ MXene quantum dots as promising nanofluorophores for metal ions sensing and fluorescence imaging. Sensor. Actuator. B. Chem. 309, 127735. doi:10.1016/j.snb.2020.127735

Yang, L., Song, Y. H., and Wang, L. (2020b). Multi-emission metal-organic framework composites for multicomponent ratiometric fluorescence sensing: recent developments and future challenges. J. Mater. Chem. B. 8, 3292-3315. doi:10.1039/c9tb01931f

Yang, Y., Wang, Y. Z., Feng, Y., Song, X. R., Cao, C., Zhang, G. L., et al. (2020c). Three isostructural $\mathrm{Eu}^{3+} / \mathrm{Tb}^{3+}$ co-doped MOFs for wide-range ratiometric temperature sensing. Talanta. 208, 120354. doi:10.1016/j.talanta.2019.120354

Yang, Z. R., Wang, M. M., Wang, X. S., and Yin, X. B. (2017). Boric-acid-functional lanthanide metal-organic frameworks for selective ratiometric fluorescence detection of fluoride ions. Anal. Chem. 89, 1930-1936. doi:10.1021/acs. analchem.6b04421

Yin, H. Q., Wang, X. Y., and Yin, X. B. (2019). Rotation restricted emission and antenna effect in single metal-organic frameworks. J. Am. Chem. Soc. 141, 15166-15173. doi:10.1021/jacs.9b06755
Yin, H. Q., and Yin, X. B. (2020). Metal-organic frameworks with multiple luminescence emissions:Designs and applications. Acc. Chem. Res. 53, 485-495. doi:10.1021/acs.accounts.9b00575

Yu, L., Zheng, Q. T., Wang, H., Liu, C. X., Huang, X. Q., and Xiao, Y. X. (2020). Double-color lanthanide metal-organic framework based logic device and visual ratiometric fluorescence water microsensor for solid pharmaceuticals. Anal. Chem. 92, 1402-1408. doi:10.1021/acs.analchem.9b04575

Yu, M. K., Xie, Y., Wang, X. Y., Li, Y. X., and Li, G. M. (2019). Highly water-stable dye@Ln-MOFs for sensitive and selective detection toward antibiotics in water. ACS Appl. Mater. Interfaces 11, 21201-21210. doi:10.1021/acsami.9b05815

Yue, D., Huang, Y. K., Zhang, L., Jiang, K., Zhang, X., Cui, Y. J., et al. (2018). Ratiometric luminescence sensing based on a mixed $\mathrm{Ce} / \mathrm{Eu}$ metal-organic framework. J. Mater. Chem. C. 6, 2054-2059. doi:10.1039/c7tc05309f

Yue, D., Wang, Y. Y., Chen, D., and Wang, Z. L. (2020). Solvent triggering structural changes for two terbium-based metal-organic frameworks and their photoluminescence sensing. Chem Comm. 56, 4320-4323. doi:10.1039/d0cc00353k

Zeng, X. L., Hu, J., Zhang, M., Wang, F. L., Wu, L., and Hou, X. D. (2020a). Visual detection of fluoride anions using mixed lanthanide metal-organic frameworks with a smartphone. Anal. Chem. 92, 2097-2102. doi:10.1021/acs.analchem.9b04598

Zeng, X. L., Long, Z., Jiang, X. F., Zhang, Y. J., Liu, Q., Hu, J., et al. (2020b). Single bimetallic lanthanide-based metal-organic frameworks for visual decoding of a broad spectrum of molecules. Anal. Chem. 92, 5500-5508. doi:10.1021/acs.analchem.0c00324

Zhang, C., Li, X., Wei, W., and Chen, Z. B. (2020). Lanthanide ions as sensor elements based sensor array for colorimetric identification of antioxidants. Sensor. Actuator. B. Chem. 305, 127532. doi:10.1016/j.snb.2019.127532

Zhang, Y. H., Li, B., Ma, H. P., Zhang, L. M., Jiang, H., Song, H., et al. (2016a). A nanoscaled lanthanide metal-organic framework as a colorimetric fluorescence sensor for dipicolinic acid based on modulating energy transfer. J. Mater. Chem. C. 4, 7294-7301. doi:10.1039/c6tc01022a

Zhang, Y. H., Li, B., Ma, H. P., Zhang, L. M., and Zheng, Y. X. (2016b). Rapid and facile ratiometric detection of an anthrax biomarker by regulating energy transfer process in bio-metal-organic framework. Biosens. Bioelectron. 85, 287-293. doi:10.1016/j.bios.2016.05.020

Zhang, Y. M., Yuan, S., Day, G., Wang, X., Yang, X. Y., and Zhou, H. C. (2018). Luminescent sensors based on metal-organic frameworks. Coord. Chem. Rev. 354, 28-45. doi:10.1016/j.ccr.2017.06.007

Zhao, D., Yue, D., Jiang, K., Zhang, L., Li, C. X., and Qian, G. D. (2019). Isostructural $\mathrm{Tb}^{3+} / \mathrm{Eu}^{3+}$ co-doped metal-organic framework based on pyridine-containing dicarboxylate ligands for ratiometric luminescence temperature sensing. Inorg. Chem. 58, 2637-2644. doi:10.1021/acs. inorgchem. 8 b03225

Zhao, Y. F., and Li, D. (2020). Lanthanide-functionalized metal-organic frameworks as ratiometric luminescent sensors. J. Mater. Chem. C. 8, 12739-12745. doi:10.1039/D0TC03430D

Zheng, X. B., Fan, R. Q., Song, Y., Wang, A. N., Xing, K., Du, X., et al. (2017). A highly sensitive turn-on ratiometric luminescent probe based on postsynthetic modification of $\mathrm{Tb}^{3+} @ \mathrm{Cu}-\mathrm{MOF}$ for $\mathrm{H}_{2} \mathrm{~S}$ detection. J. Mater. Chem. C. 5, 9943-9951. doi:10.1039/c7tc02430d

Conflict of Interest: The authors declare that the research was conducted in the absence of any commercial or financial relationships that could be construed as a potential conflict of interest.

Copyright () 2021 Sun, Gao, Du, Zhou and Chen. This is an open-access article distributed under the terms of the Creative Commons Attribution License (CC BY). The use, distribution or reproduction in other forums is permitted, provided the original author(s) and the copyright owner(s) are credited and that the original publication in this journal is cited, in accordance with accepted academic practice. No use, distribution or reproduction is permitted which does not comply with these terms. 